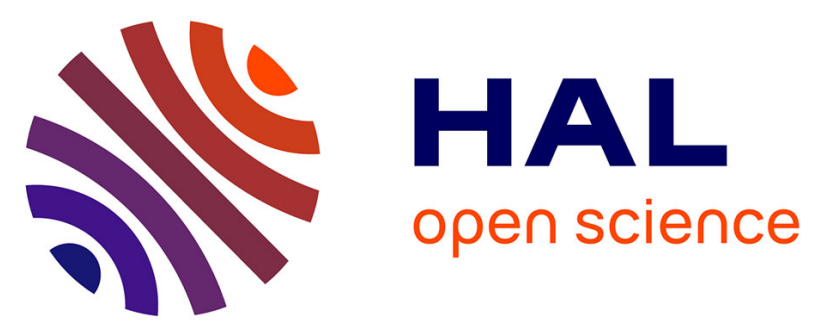

\title{
Effect of mechanical stress on different core loss components along orthogonal directions in electrical steels
}

\author{
A. Baghel, J. Blumenfeld, L. Santandrea, G. Krebs, L. Daniel
}

\section{- To cite this version:}

A. Baghel, J. Blumenfeld, L. Santandrea, G. Krebs, L. Daniel. Effect of mechanical stress on different core loss components along orthogonal directions in electrical steels. Electrical Engineering, 2019, 101 (3), pp.845-853. 10.1007/s00202-019-00827-4 . hal-02504804

\section{HAL Id: hal-02504804 \\ https://hal-centralesupelec.archives-ouvertes.fr/hal-02504804}

Submitted on 11 Mar 2020

HAL is a multi-disciplinary open access archive for the deposit and dissemination of scientific research documents, whether they are published or not. The documents may come from teaching and research institutions in France or abroad, or from public or private research centers.
L'archive ouverte pluridisciplinaire HAL, est destinée au dépôt et à la diffusion de documents scientifiques de niveau recherche, publiés ou non, émanant des établissements d'enseignement et de recherche français ou étrangers, des laboratoires publics ou privés. 


\section{Electrical Engineering \\ Effect of mechanical stress on different core loss components along orthogonal directions in electrical steels \\ --Manuscript Draft--}

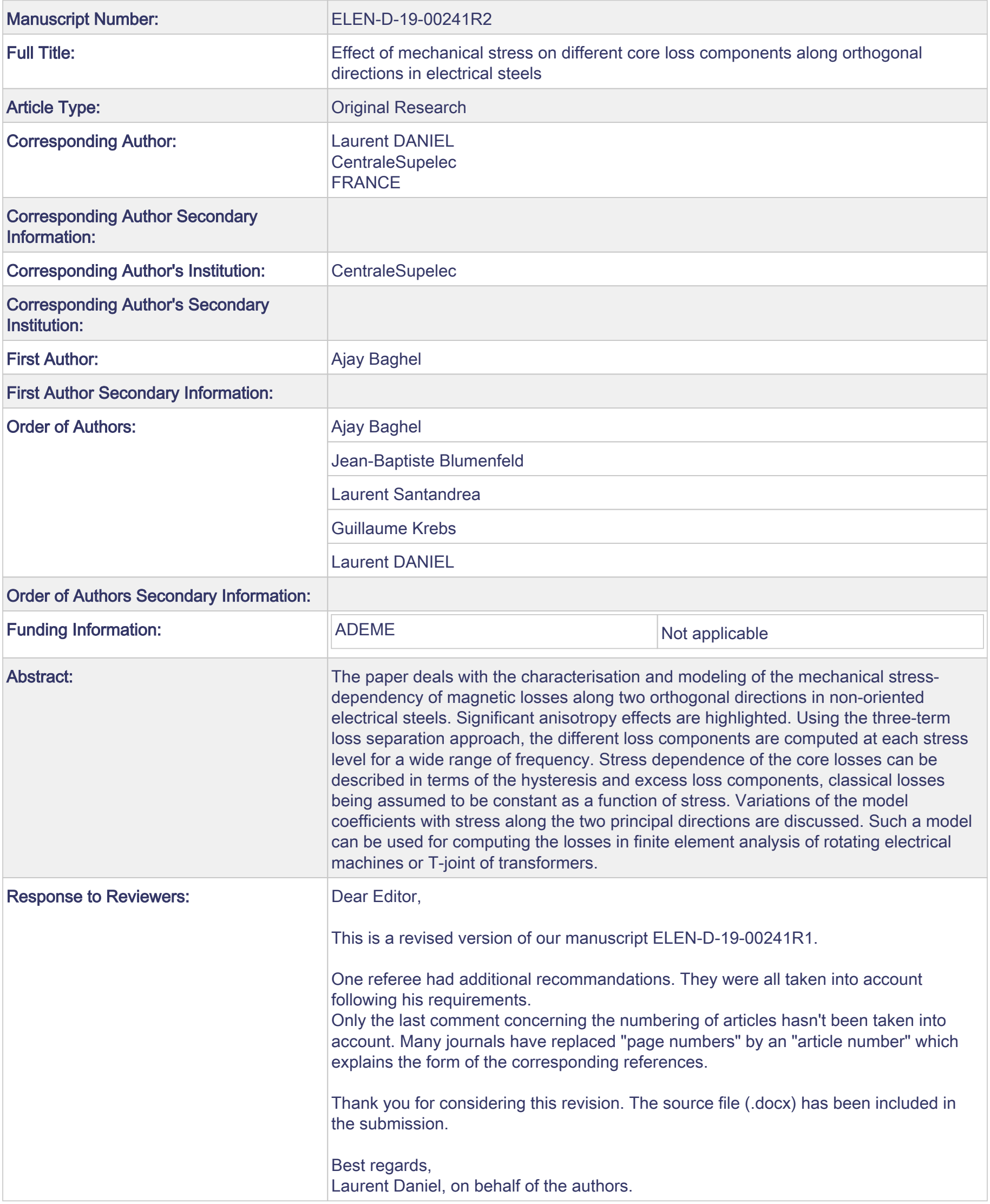




\title{
Effect of mechanical stress on different core loss components along orthogonal directions in electrical steels
}

\author{
A. P. S. Baghel ${ }^{1}$, J.B. Blumenfeld ${ }^{2}$, L. Santandrea ${ }^{1}$, G. Krebs ${ }^{1}$, and L. Daniel ${ }^{1}$ \\ ${ }^{1}$ GeePs | Group of electrical engineering - Paris, UMR CNRS 8507 CentraleSupélec, Univ. Paris-Sud, Univ. Paris-Saclay, \\ Sorbonne Université, 3-11 rue Joliot-Curie, Gif-sur-Yvette, F-91192, France. \\ ${ }^{2}$ Renault, 1 avenue du Golf, 78288 Guyancourt, France
}

E-mail: ajaybaghel3@gmail.com; jean-baptiste.blumenfeld@ renault.com; laurent.santandrea@geeps.centralesupelec.fr; guillaume.krebs@geeps.centralesupelec.fr; laurent.daniel@ centralesupelec.fr.

\begin{abstract}
The paper deals with the characterisation and modeling of the mechanical stress-dependency of magnetic losses along two orthogonal directions in non-oriented electrical steels. Significant anisotropy effects are highlighted. Using the three-term loss separation approach, the different loss components are computed at each stress level for a wide range of frequency. Stress dependence of the core losses can be described in terms of the hysteresis and excess loss components, classical losses being assumed to be constant as a function of stress. Variations of the model coefficients with stress along the two principal directions are discussed. Such a model can be used for computing the losses in finite element analysis of rotating electrical machines or T-joint of transformers.
\end{abstract}

Keywords: Core loss, loss separation approach, mechanical stress, electrical steels

\section{Introduction}

The design of electrical machines for more compact and high-speed systems demands reduction in size and weight. On the other hand, these systems are very sensitive to mechanical stresses which can be inherited from manufacturing stage (cutting, stacking, shrink fitting, ...) or appear in operating conditions (magnetic forces, centrifugal forces, ...) [1-2]. The manufacturing processes are usually associated to residual stresses within the material which can significantly affect the material behaviour, even far from plastically deformed areas. Mechanical stresses appearing in use usually remain within the elasticity range and also affect strongly the material behaviour [2-3].

Numerous experimental studies on the influence of mechanical stress on the magnetic properties are reported in the literature [4-8]. Some of them show the influence of mechanical stress on magnetic properties using the direct observation of magnetic domains in grain-oriented materials [5-6]. Effects of mechanical stress in non-oriented materials were reported recently [8] based on similar interpretation as for grain-oriented materials. It shows that the compressive stress monotonically deteriorates magnetic properties as compression stress disfavours the magnetic easy axis along the rolling direction. On the other hand, tensile stress first improves the magnetic properties and then after a certain value of applied stress, magnetic properties continuously deteriorate. This can be explained from a microstructural interpretation since the applied stress initally favours the magnetic easy axis and at higher stress values demagnetizing field may be dominant [6]. Other results on the effect of tensile stress and plastic deformation in electrical steels have been reported in [7].

These effects of stress are observed in particular on magnetic permeability and magnetic losses in core materials of electrical machines [9]. Indeed, ferromagnetic materials exhibit a strongly coupled magneto-mechanical behavior [1011]. Therefore, the coupled behavior needs to be taken into consideration in modern machine-design tools. An accurate model for the description of magneto-mechanical effects is a 
prerequisite for such a tool. Numerous attempts have been reported in the literature which consider magneto-mechanical coupled behavior into finite-element (FEM) simulations [1214]. However, the most common models are usually uncoupled and often consider uniform non-linear anhysteretic magnetic behavior in Finite Element Analysis (FEA) simulations and iron losses to be computed at the postprocessing stage [15-17]. This approach is also known as $a$ posteriori approach. A significant number of papers on the magneto-elastic constitutive law for ferromagnetic materials can be found in the literature [18-22]. However, the numerical implementation of these models in FEM (also known as intrinsic approaches) is not a straightforward task as it takes huge computation time and poses convergence issues as well. Hence, the former is generally a preferred choice if its accuracy is within an acceptable range. For such an analysis, anhysteretic magnetic and iron loss behavior should consider the influence of mechanical stresses.

According to statistical loss theory, the core losses can be classified into three components, namely, hysteresis, classical eddy current and excess losses [23]. A number of loss formula based on the loss separation approach has been reported in the literature [24-27], which can be applied to compute core losses at post-processing stage in FEM analyses of electrical machines.

A significant number of studies are reported on the description of stress effects on the core losses using the loss separation approach [28-32]. The behaviour of loss components under applied mechanical stress have been studied and the loss coefficients modeled using the exponential functions [28] or second -order polynomial [30]. Although the effect of compressive and tensile stress on the iron losses has been studied extensively in the literature [9,11,28-32], most of the existing attempts of loss modeling are limited to the rolling direction (RD) only [28,30,32]. The effect of stress on magnetic losses in other than rolling direction is rarely addressed in the literature [3]. However, even if the anisotropy of non-oriented iron-silicon steels is known to be moderate, significant differences can be observed in the effect of stress on core losses along the rolling and transverse (TD) directions. This anisotropy is illustrated in Fig. 1 for a non-oriented material (M235-35A) at $1 \mathrm{~T}$ and 50 $\mathrm{Hz}$ subjected to uniaxial tensile and compressive stress.

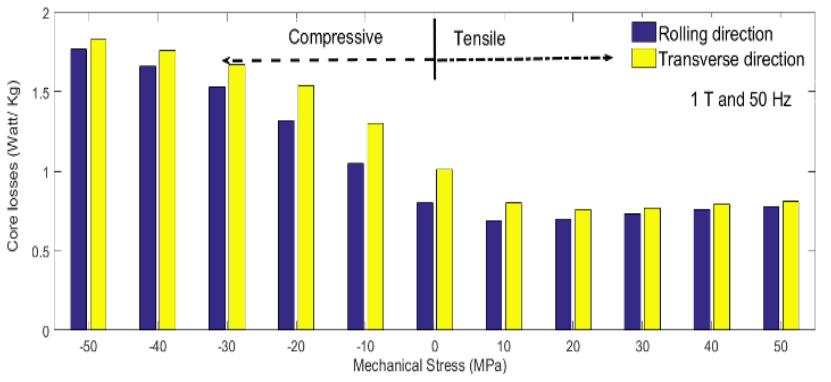

Figure 1: Core losses along two principal directions (rolling - RD and transverse - TD) under applied uniaxial mechanical stress. The stress is applied along the magnetic field direction

The $\%$ difference in core losses along RD and TD at different stress levels (compression and tension) is depicted in Fig. 2 (a). The maximum difference between the losses in the two orthogonal directions occurs at zero stress condition and decreses as mechanical stress increases [3]. The application of stress then tends to uniformise the behaviour and hence reduce the anisotropy. This can be connected to the modification in the domain configuration which becomes more and more dominated by stress effects as the level of stress increases [8]. The difference in the core loss with respect to zero stress condition is shown in Fig. 2(b). Significant changes can be observed in both principal directions as the applied stress increases. It is evident that compression significantly increase the magnetic losses while tension has a more moderate, and initially beneficial, effect.

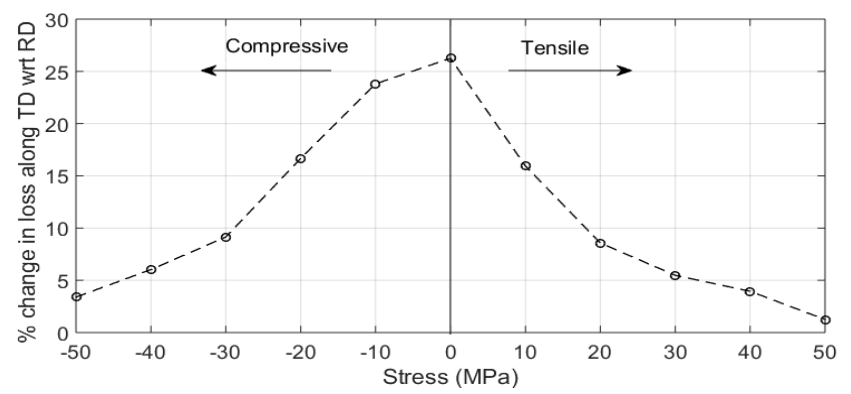

(a)

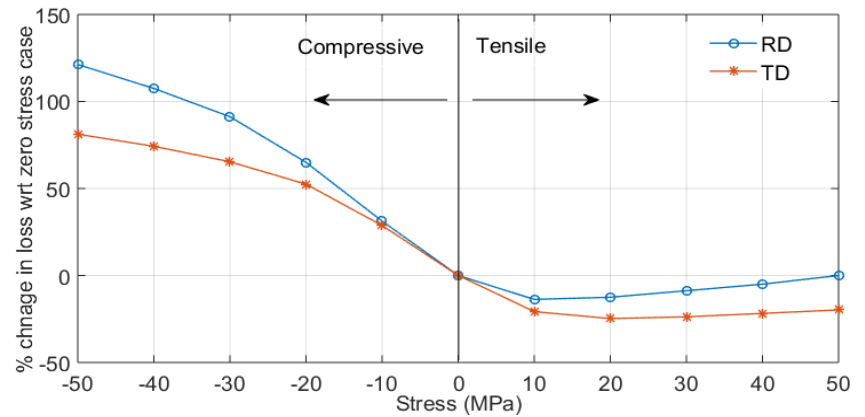

(b)

Figure 2: Effect of mechanical stress on the core losses along two principal directions at $1 \mathrm{~T}, 50 \mathrm{~Hz}$ (a) \% change in loss along TD wrt $\mathrm{RD}$ at different stress levels, (b) \% change in loss wrt to unstressed condition along RD and TD

Loss properties being one of the key-point for design and development of high power density and efficient machines, there is a need for the development of loss models considering the effects of stress. Moreover, the magnetic flux does not remain along a single direction, especially, in joint and teeth regions of electrical machines. Therfore, the anisotropic feature of the core losses in electrical steels should be considered for an accurate loss computation in electrical machines [33]. This is why it is useful to describe loss behavior in electrical steels along different directions. This 
can help designers and researchers in the development of efficient loss models. Furthermore, studies on magnetomechanical effects are vital for optimization of electrical machines, determination of lifetime performance, and material choice.

This paper investigates the effect of stress on the core losses in two orthogonal directions. The paper also presents an approach to model these effects in both principal directions. The suggested approach is based on the loss separation and investigates the effects of mechanical stress using a frequency domain loss model. As evident from Fig 1, compressive stresses affect the core loss more significantly than the tensile stress. Therefore, this paper focuses on the modeling of compressive stress-effects on the core losses along the two principal directions. The paper also discusses variations of loss components with stress. The loss separation approach is discussed in section 2 and mechanical stress-dependency of loss model parameters is given in section 3 for both rolling (RD) and transverse (TD) directions.

\section{Measurements and Modelling of Core Losses}

\subsection{Experimental setup}

The measurements of hysteresis loops and corresponding losses are carried out on samples (non-oriented electrical steel; grade-M235-35A) which are cut at two angles of $0^{\circ}$ and $90^{\circ}$ with respect to RD. The measurements are performed on a single sheet tester (SST), with tension and compression mechanical unit as shown in Fig. 3. The magnetic measurement system (SST) is a BROCKHAUS-MPG $200 \mathrm{D}$ device. The measurements are made under standard pure sinusoidal waveforms of induction levels $(0.2-1.5 \mathrm{~T})$ for a frequency range of $10 \mathrm{~Hz}-2 \mathrm{kHz}$. Each measurement is carried out twice and the results are averaged. Stress is applied through a pneumatic tension and compression unit. Given the dimensions of the sample (standard stripe, $30 \mathrm{~mm}$ width and 0.35 thickness), the pneumatic unit is capable of applying uniaxial stresses up to $70 \mathrm{MPa}$. The resolution on stress is approximately $3.5 \mathrm{MPa}$. During compression, a wedge is placed on each side of the sample in order to avoid buckling. This allows loading the sample up to $50 \mathrm{MPa}$ in compression.

From the measured B-H curves, total core losses at each frequency and stress level are calculated by computing the area of hysteresis loops. The separation of total core loss into three components viz., hysteretic, classic and excess terms, is a common practice for loss description in thin ferromagnetic laminations [34].

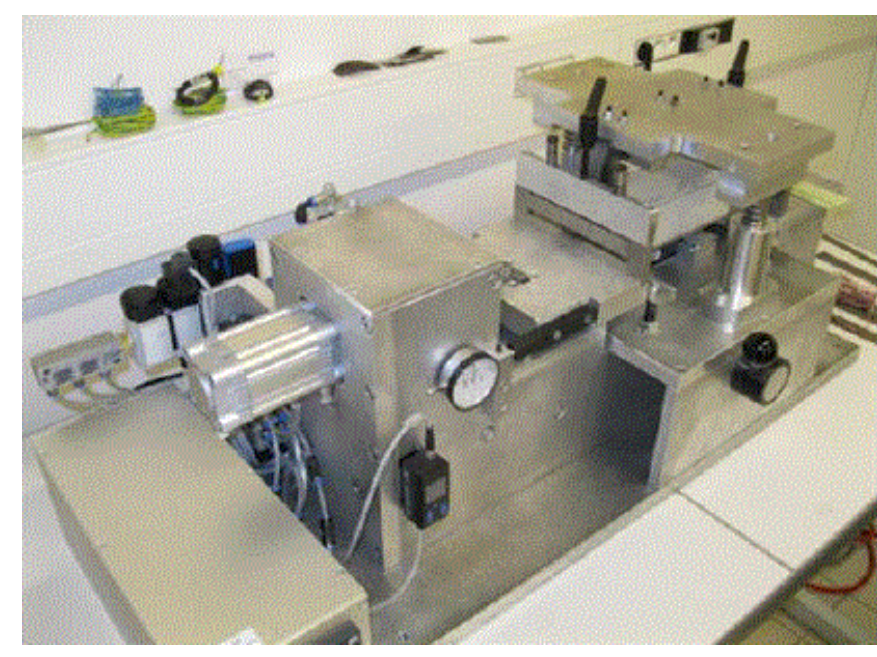

Figure 3: Single sheet tester with the mechanical unit for stresses (Source: RENAULT-SAS, Guyancourt, France)

\subsection{Loss separation model}

The loss mechanisms associated to the magnetization process is a complex phenomenon that is still far from being understood [35]. A standard physics based loss computational model is yet to achieve. Empirical approaches can be used to describe iron losses in an approximate manner [36]. The core losses, from an engineering viewpoint, can be separated in three components namely, the hysteresis, classical eddy current, and excess or anomalous losses [9,34]. In recent years a number of advances have been proposed in the loss separation models [25] in order to consider harmonics or minor loops [15, 16, 27].

For electrical steels, the decomposition of the total loss into three components is a common practice. The total loss can be rewritten as [34]:

$$
P_{\mathrm{tot}}=P_{\mathrm{h}}+P_{e c}+P_{\mathrm{ex}}
$$

where $P_{\text {tot }}$ is the total core loss $(\mathrm{W} / \mathrm{kg}), P_{\mathrm{h}}$ is the hysteresis loss, $P_{e c}$ is the eddy current loss, and $P_{e x}$ is the excess or anomalous losses.

The static hysteresis losses can be explained through the irreversible motion of domain walls [37]. The losses at very low frequency can be referred to as the quasi-static or dc hysteresis loss which is computed by the area of DC hysteresis loops. This component is strongly related to the domain microstructure of materials [35]. It can be represented as:

$$
P_{h}=k_{h} \widehat{B}^{\alpha} f
$$

Here, $k_{h}$ and $\alpha$ are material constants which depend on the domain structure and crystalline orientations of materials.

The classical eddy current loss depends on the supply frequency, sample thickness, and conductivity of laminations under the assumption of homogeneity of the material [34]. Conductivity of the sample is usually assumed to be isotropic and independent of stress. The eddy current losses can be calculated using the following equation: 


$$
P_{e c}=k_{e c} \widehat{B}^{2} f^{2}
$$

Here, $k_{e c}$ is a coefficient which depends on the geometrical dimensions and the chemical composition of the material. $\widehat{B}$ and $f$ are the peak inducton value and frequency respectively.

An excess power loss that depends on domain configuration and microscopic eddy currents is modelled as [35]:

$$
P_{\text {exc }}=k_{e x} \widehat{B}^{1.5} f^{1.5}
$$

Here, $k_{e x}$ is a material coefficient and it depends on the domain structure in the material.

According to conventional models, the eddy current loss, also referred to as the classical loss, can be calculated directly as [24]:

$$
k_{e c}=\frac{(\pi d)^{2}}{6 \rho D}
$$

Here, $d, \rho$, and $D$, are the sheet thickness $(m)$, resistivity $(\Omega$ $\mathrm{m})$ and mass density $\left(\mathrm{kg} / \mathrm{m}^{3}\right)$ of the core material respectively. Due to inherent limitations of conventional models (with 4 coefficients to identify), the accuracy of loss computation may not be within acceptable limits for a wide range of frequencies and induction levels. On contrary, an effective loss model is essential for accurate design of electrical machines, where varying magnetic loadings and flux-weakening at high speeds in variable-speed machines are often encountered [25]. Therefore, numerous empirical models have been proposed for loss evaluation over wide ranges of frequencies and induction levels $[25,36]$.

A three-term loss model using a modified Steinmetz formula has been given as [36]:

$$
P_{\text {tot }}=k_{h} \widehat{B}^{\alpha} f+k_{e c} \widehat{B}^{2} f^{2}+k_{e x} \widehat{B}^{1.5} f^{1.5}
$$

In this approach, the measured loss data of a particular material over a range of frequency and induction levels is fitted to a loss prediction formula and the unknown parameters of the model are determined based on a data fitting algorithm as discussed in [24]. Moreover, the loss model (6) has been further modified in order to consider dynamic hysteresis and skin-effects at higher frequencies as [38]:

$$
P_{\text {tot }}=k_{h} \widehat{B}^{\left(\alpha_{0}+\alpha_{1} B+\alpha_{2} B^{2}\right)} f+k_{e c} \widehat{B}^{2} f^{2}+k_{e x} \widehat{B}^{1.5} f^{1.5}
$$

Here the parameters $\left(k_{e c}, k_{e x}\right)$ varies with induction levels and can be represented as a function of B [36]. However, hysteresis loss coefficients $\left(k_{\mathrm{h}}, \alpha\right)$ are frequency-dependent. Moreover, the hysteresis exponent coefficient $(\alpha)$ is represented as a second order polynomail of $B$ which considers the skin-effect and dynamic hysteresis [38]. The algorithm, however, is simple and valid for a frequency range from static up to $2 \mathrm{kHz}$ [36].

\subsection{Loss coefficient identification}

The loss coefficients $\left(k_{e c}, k_{e x}, k_{\mathrm{h}}\right.$, and $\alpha$ ) can be obtained using the algorithms presented in $[24,36]$. By dividing the total losses by the frequency, Eq. (7) can be rewritten as:

$$
P_{\text {tot }} / f=a+b f+c \sqrt{f}
$$

Here, the coefficients $a, b$, and $c$ are represented as:

$$
\begin{aligned}
& a=k_{h} \widehat{B}^{\alpha} \\
& b=k_{e c} \widehat{B}^{2} \\
& c=k_{e x} \widehat{B}^{1.5}
\end{aligned}
$$

The measured loss values at least for three frequencies are required for determining three coefficients $a, b$ and $c$ in (8) at any induction level. From (8) and (9), the eddy current $\left(k_{e c}\right)$ and excess loss coefficients $\left(k_{e x}\right)$ can easily be computed. These coefficients show a significant variation with induction levels but no dependence on frequency [24]. A second order polynomial is used to fit these variations as:

$$
\begin{aligned}
& k_{e c}=k_{e c 0}+k_{e c 1} B+k_{e c 2} B^{2} \\
& k_{e x}=k_{e x 0}+k_{e x 1} B+k_{e x 2} B^{2}
\end{aligned}
$$

In order to identify the hysteresis loss coefficients $\left(k_{\mathrm{h}}, \alpha\right)$, Eq. (8) can be rewritten using the logarithmic operator as:

$\log a=\log k_{h}+\left(\alpha_{0}+\alpha_{1} \cdot B\right) \cdot \log B$

The hysteresis exponent coefficient $(\alpha)$ is taken in this work as a linear function of $\mathrm{B}$ in order to reduce the unknown parameters and required measured data for parameter identification. For three unknowns, Eq. (11) needs the loss data at least for three induction levels. The coefficient $a$ represents the ratio of hysteresis loss and frequency and it can be computed using (8) by substituting the values of $b$ and $c$ which are obtained from (9).

\subsection{Modelling of core losses using the loss separation theory}

The loss model coefficients under no applied stress are identified using the measured loss data at three induction levels (here 0.2, 0.7 and $1.5 \mathrm{~T}$ ) and three frequencies (here 50, 200 , and $800 \mathrm{~Hz}$ ) for the frequency range $10 \mathrm{~Hz}-1 \mathrm{kHz}$. The obtained loss coefficients are given in Table 1.

Table 1: Determined loss coefficients

\begin{tabular}{|c|c|}
\hline Parameters & Identified Values (unitless) \\
\hline$k_{h}$ & 0.0121 \\
\hline$\alpha\left(\alpha_{0}, \alpha_{1}\right)$ & $\alpha_{0}=1.6829 ;$ \\
$\alpha_{1}=0.4050$ \\
\hline$k_{e c}\left(k_{e c 0}, k_{e c 1}, k_{e c}\right)$ & $k_{e c 0}=3.9305 \times 10^{-5} ; k_{e c 1}=-4.4837 \times 10^{-4} ;$ \\
& $k_{e c 2}=3.9840 \times 10^{-5}$ \\
\hline$k_{e x}\left(k_{e x 0}, k_{e x 1}, k_{e x 2}\right)$ & $k_{e x 0}=6.2871 \times 10^{-5} ; k_{e x 1}=0.0013 ;$ \\
& $k_{e x 2}=-8.4956 \times 10^{-4}$ \\
\hline
\end{tabular}


The computed and measured losses are shown to be in close agreement at various induction levels and for a wide frequency range as depicted from Figs 4(a)-(c). The magnitude of maximum error for a frequency has not crossed beyond $12 \%$ in this analysis as can be seen from Fig. 4(b).

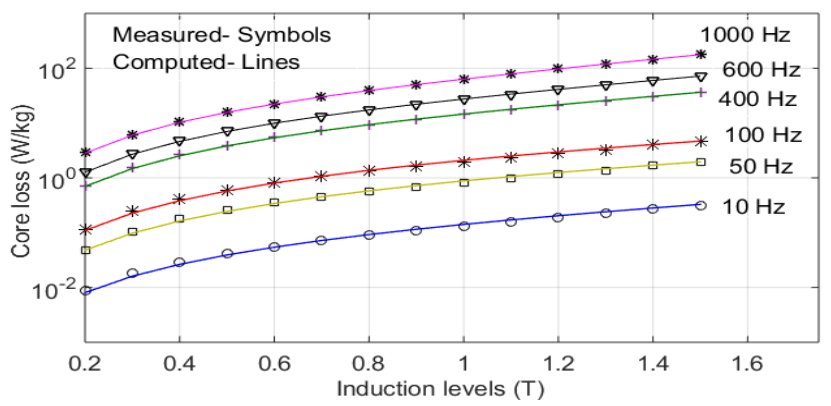

(a)

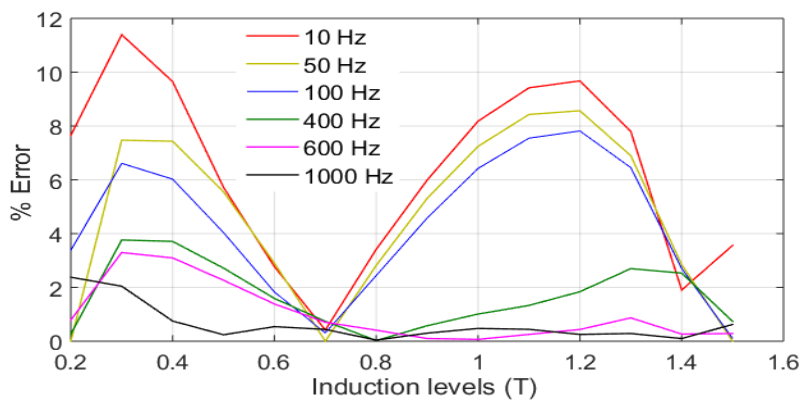

(b)

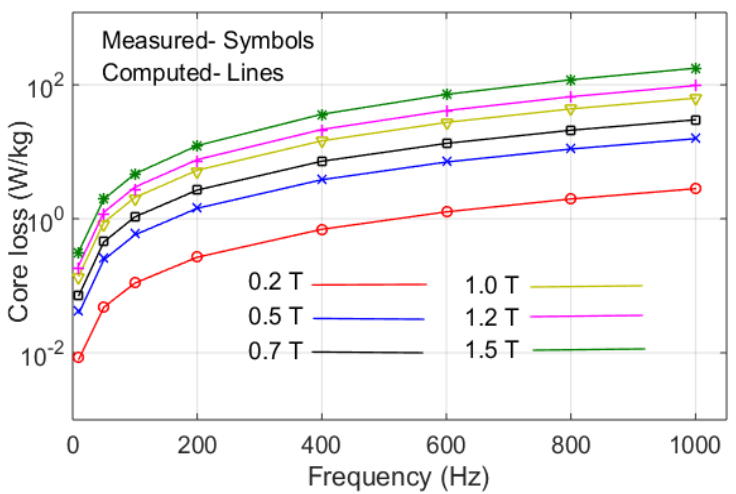

(c)

Figure 4: computed and measured core loss: (a) losses vs induction (b) \% Error vs induction levels at different frequencies (c) losses vs frequency (the loss values at $0.2,0.7$, and $1.5 \mathrm{~T}$ and at $50 \mathrm{~Hz}, 200 \mathrm{~Hz}$, and $800 \mathrm{~Hz}$ are used in the identification algorithm)

The eddy current and excess loss parameters can then be represented as a second-degree polynomial function of induction (B; as given in Eq. 10) to get better accuracy over a wide range of induction values. Variations of these parameters $\left(k_{e c}, k_{e x}\right)$ and different loss components with induction levels are shown in Figs 5(a)-(b). Variations of the two loss coefficients $\left(k_{e c}, k_{e x}\right)$ with induction levels are complementarylike to each other. Moreover, the excess loss is the maximum between $0.6 \mathrm{~T}-0.8 \mathrm{~T}$ and then decreases at higher induction levels. Eeddy current losses are minimum between 0.6 T-0.8 $\mathrm{T}$ and then start to increase at higher induction values.
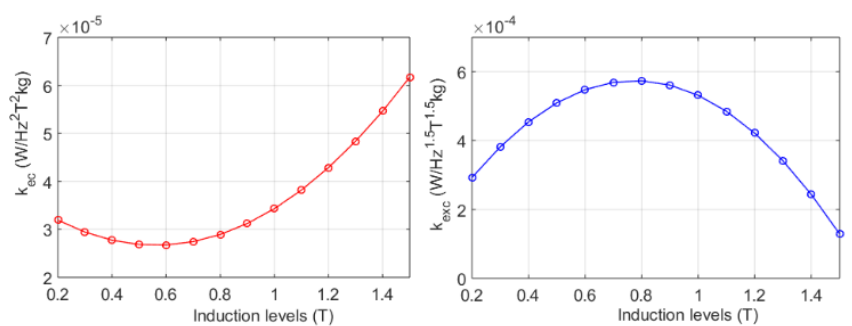

(a)
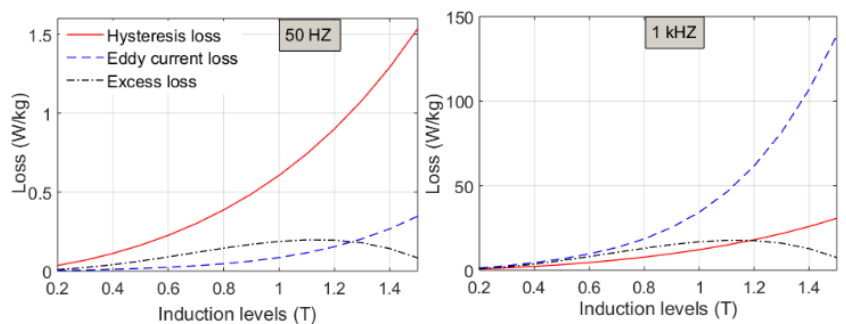

(b)

Figure 5: Variation of (a) loss coefficients with induction levels (b) loss components with induction levels at different frequencies

It is shown that the suggested model (7) can satisfactorily describe the loss behavior of this material. It is used in the next section to study the effect of mechanical stresses on the core losses in the same material.

\section{Investigation of loss behaviour along the rolling and transverse directions}

\subsection{Loss-stress behavior along the rolling and transverse directions}

In order to investigate the stress effects on the losses along the two orthogonal directions, the loss separation approach is used to compute each component along these directions at different stress levels. The loss separation is performed along $\mathrm{RD}$ and TD to investigate the behavior of three losscomponents at different stress levels. The classical loss component is assumed to be independent of stress and orientation. Hence, this component is assumed to remain the same for all directions and stress levels and it can be computed from RD measurement at zero stress level. The hysteresis and excess loss components vary with the mechanical stress as they depend on the domain configuration of the material. Therefore, two loss coefficients need to be identified (hysteresis and excess loss coefficient). They can be determined using the measured loss data (at three induction levels (here $0.2 \mathrm{~T}, 0.7 \mathrm{~T}$, and $1.5 \mathrm{~T}$ ) but only for two frequencies (here 50 and $800 \mathrm{~Hz}$ )) for each stress. 
The computed loss components and the measured core loss

(a)
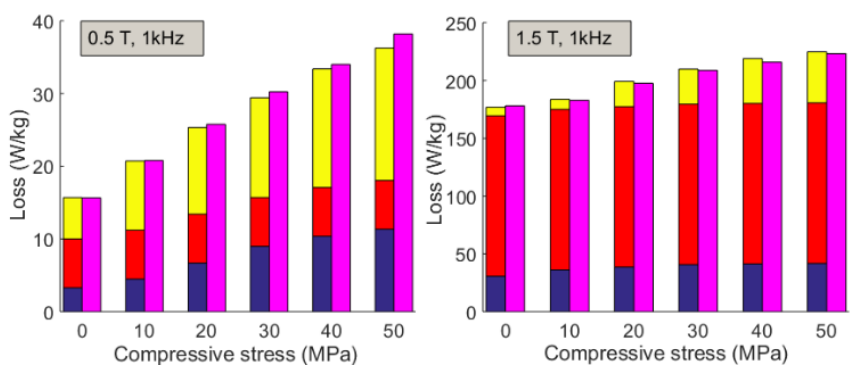

(b)

Figure 6: Computed loss components and measured core losses with compressive stress along RD

The same approach is carried out on the core loss data along TD. It is reminded here that the eddy current loss will remain the same at each stress level and whatever the direction of the applied magnetic field. Therefore, parameter identification is performed at the same induction levels but only for two frequencies (here $50 \mathrm{~Hz}$ and $800 \mathrm{~Hz}$ ) as used for RD at nonzero stress levels. Computed and measured losses along TD at different induction levels and frequencies are shown in Figs 7 (a)-(b).
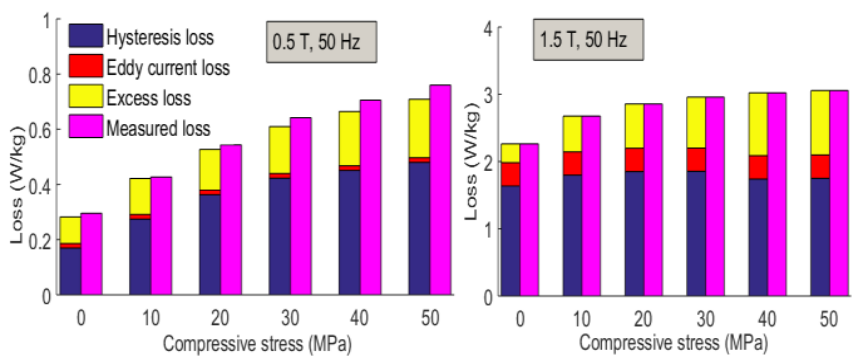

(a)
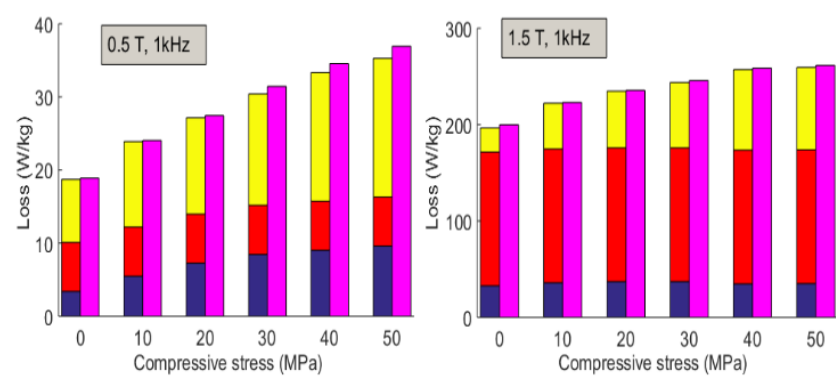

(b)

Figure 7: Computed loss components and measured core losses with compressive stress along TD

Using the identified parameters, the model can be used to predict core losses at other induction levels and frequency (e.g. $1.0 \mathrm{~T}, 2 \mathrm{kHz}$ ) with reasonable accuracy as shown in Fig. 8. The magnitude of maximum error for a frequency has not crossed beyond $15 \%$ in this analysis. The accuracy of the analysis can be further improved by using higher order polynomial for $k_{e c}(\mathrm{~B}), k_{e x}(\mathrm{~B})$, and $\alpha(\mathrm{B})$ as discussed in [25].

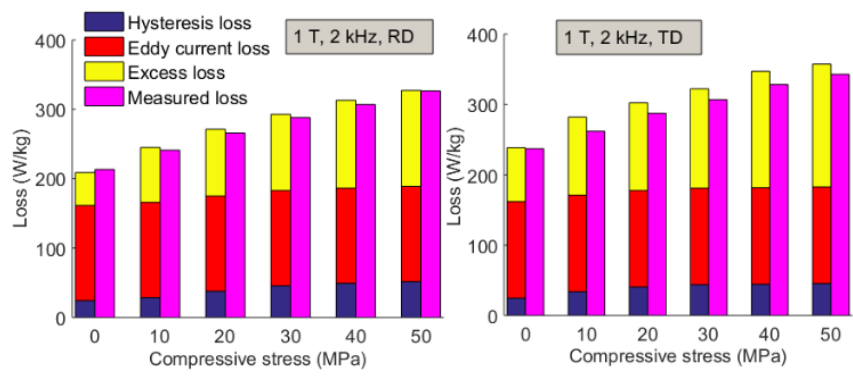

Figure 8: Predicted and Measured losses along RD and TD

\subsection{Results and discussions}

The effects of microstructural features (crystallographic texture, grain size, dislocation densities, residual stresses, etc.) on the core losses can be described in terms of $k_{e x}$ and $k_{h}$ [35]. These parameters are a signature of the effect of mechanical stress on the microstructure and magnetic properties of electrical steels [29]. Hysteresis and excess loss variations with compressive stress show similar trends as shown in Figs 9 (a)-(b).
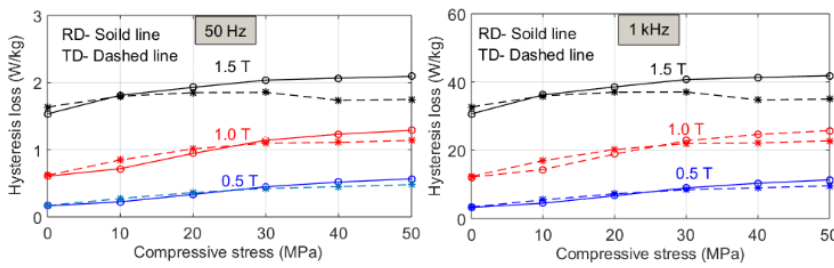

(a) 

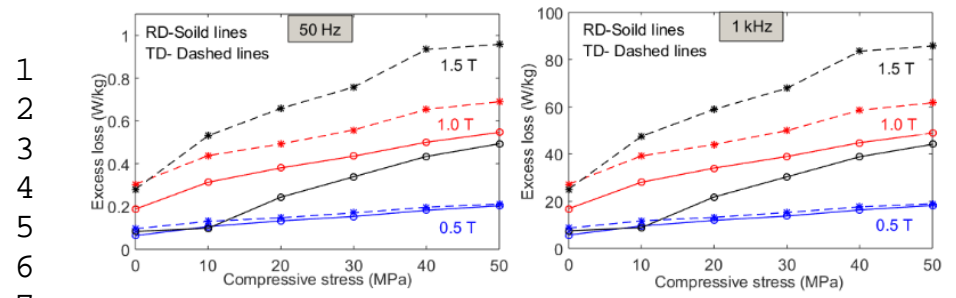

(b)

Figure 9: Variation of loss components with compressive stress at different frequencies and induction levels (blue) $=0.5 \mathrm{~T} ;$ red $=1.0$ $\mathrm{T}$; black $=1.5 \mathrm{~T}$; circle (solid lines $)=\mathrm{RD}$; cross $($ dashed lines $)=\mathrm{TD})$

$\left(\right.$ a) hysteresis component $\left(P_{\text {hyst }}\right)(b)$ excess loss component $\left(P_{\text {exc }}\right)$

It can be observed from Fig. 9 that both hysteresis and excess components show similar trends (monotonocally increasing with stress) at different frequency and induction levels. The excess loss component for TD, as shown in Fig. 9 (b), is higher than that of RD up to $50 \mathrm{MPa}$. On the other hand, the hysteresis loss component along TD is higher than that of RD up to a certain stress level and beyond this stress level it becomes lower than RD. The obtained results are in line with those reported in [3] which uses although a two-term loss formula. In order to investigate the stress-dependency of loss components, variations of loss coefficients with stress are shown in Figs 10 (a)-(b). Parameters for hysteresis $\left(k_{\mathrm{h}}\right)$ and excess $\left(k_{\mathrm{ex}}\right)$ losses increase monotonically with the compression stress level. On the other hand, the hysteresis exponent coefficient $(\alpha)$ decreases as the applied stress increases. These parameter variations with stress have been modeled using exponential function [28] and second order polynomail [30] in the literature. However, these works are restricted to RD. The present work provides an approach to model stress-dependencies on loss parameters along both principal directions. The difference in the loss components for the two orthogonal directions might be attributed to the anisotropy due to the crystallographic texture of the material That is the reason why only two components (hysteresis and excess loss which depend on domain configurations) are shown to vary with respect to stress and direction as well. On the other hand, the classical eddy current loss is assumed to be constant with respect to applied stress and orientation of magnetic field. This is consistent with previous works $[28,30]$.

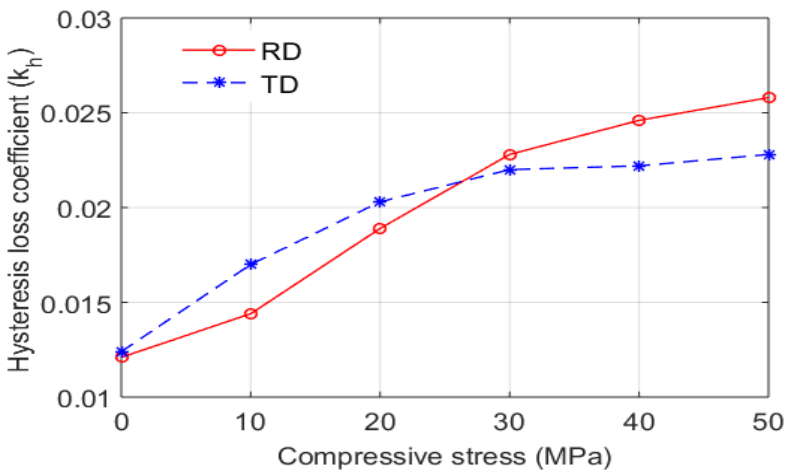

(a)
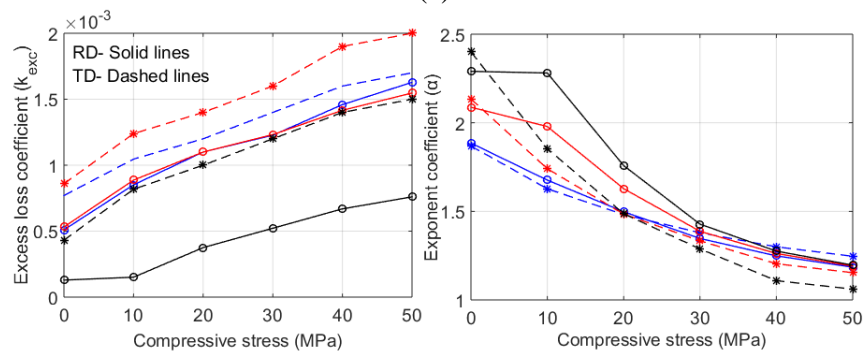

(b)

Figure 10: Variation in loss coefficient with compressive stress along RD and TD (a) $k_{\mathrm{h}}$ (b) $k_{e x}$ and $\alpha(($ blue $)=0.5 \mathrm{~T}$; red $=1.0 \mathrm{~T}$; black $=1.5 \mathrm{~T}$; circle $($ solid lines $)=\mathrm{RD}$; cross $($ dashed lines $)=\mathrm{TD})$

Variations of model parameters with stress show almost similar trends along the two directions. Therefore, it can be concluded that the loss-stress dependency shows similar trends along both the principal directions. In other words, the stress can be included in the suggested loss model representing its coefficients as a similar function of stress along both directions. The accuracy of the loss model when stress effects are included can be improved by choosing appropriate stress-dependent functions for the loss coefficients $[28,30]$. Of course, such a single dimensional approach has to be generalised to multiaxial configurations, either by using multiaxial measurement apparatus [39-41], or based on 3D modelling approaches [42-43].

\section{Conclusions}

The effect of uniaxial mechanical stress, particularly compressive stress, on iron losses is noticeable in both principal directions. The losses under $50 \mathrm{MPa}$ compression stress can be $120 \%$ higher than that under unstressed condition along RD and $85 \%$ higher along TD. Therefore, these effects should be considered in loss computations for electrical machines. It can also be observed that at higher stress levels, the effect of stress is highly detrimental to the loss performance and the difference between RD and TD vanishes. In this paper, using a modified empirical loss model, stressdependencies of the hysteresis and excess loss components along RD and TD are studied for a wide range of frequencies (from static up to $2 \mathrm{kHz}$ ) and induction levels $(0.1 \mathrm{~T}-1.5 \mathrm{~T}$ ). The eddy current loss component is assumed to be independent of mechanical stress. The variations of loss coefficients with compressive stress are investigated in the two principal directions. These variations in hysteresis and excess loss parameters against the stress level are almost similar along the two directions. It should be noted that the proposed analysis is valid only for the case of elastic mechanical loadings. The stress-loss behaviour does not show strong orientation dependencies as the stress dependencies of different loss components follow the same trends along both direction. Higher losses are nevertheless observed along TD 
compared to RD whixh is attributed to the anisotropy of the material resulting from the crystallographic texture.

The computed core losses using the suggested loss model matches with the measured total core losses along RD and TD. Such a study can be useful for a better prediction of the iron losses in rotating electrical machines [33]. Implementation of the model in FEM analysis of electrical machines is identified as a future work.

\section{Acknowledgements}

This work is part of the COCTEL project coordinated by RENAULT-SAS, Guyancourt, France and funded by ADEME (French Environment and Energy Management Agency).

\section{References}

[1] Bernard, L. and Daniel, L. (2015) Effect of stress on magnetic hysteresis losses in a switched reluctance motor: application to stator and rotor shrinking fitting: IEEE Trans Magn 517002513.

[2] Miyagi, D., Maeda, N., Ozeki, Y., Miki, K., and Takahashi, N. (2009) Estimation of iron loss in motor core with shrink fitting using FEM analysis: IEEE Trans Magn 45 1704-1707.

[3] Miyagi, D., Miki, K., Nakano, M., and Takahashi, N. (2010) Influence of compressive stress on magnetic properties of laminated electrical steel sheets: IEEE Trans Magn 46 318-321.

[4] Schneider, C.S. (2005) Effect of stress on the shape of ferromagnetic hysteresis loops: J Appl Phy 97 10E503.

[5] Perevertov, O. and Schäfer R. (2012) Influence of applied compressive stress on the hysteresis curves and magnetic domain structure of grain-oriented transverse $\mathrm{Fe}-3 \% \mathrm{Si}$ steel: $J$. Phys. D: Appl. Phys. 45135001 (11pp).

[6] Perevertov, O. and Schäfer R. (2014) Influence of applied tensile stress on the hysteresis curve and magnetic domain structure of grain-oriented Fe-3\%Si steel: J. Phys. D: Appl. Phys. 47185001 (10pp).

[7] Leuning, N., Steentjes, S., Schulte, M., Bleck, W., and Hamayer, K. (2016) Effect of elastic and plastic tensile mechanical loading on the magnetic properties of NGO electrical steels: J Magn Magn Mater 417 42-48.

[8] Perevertov, O. (2017) Influence of the applied elastic tensile and compressive stress on the hysteresis curves of $\mathrm{Fe}-3 \% \mathrm{Si}$ nonoriented steel: J Magn Magn Mater 428 223-228.

[9] Moses, A.J. (1979) Effects of applied stress on high permeability silicon-iron: IEEE Trans Magn 15 1575-1579.

[10] Cullity, B.D. and Graham, C.D. 2009 Introduction to Magnetic Materials (Wiley \& Sons: 2nd edition).

[11] Bozorth, R.M. 1951 Ferromagnetism (Van Norstand).

[12] Mohammed, O., Calvert, T., and McConnell, R. (1999) A model for magnetostriction in coupled nonlinear finite element magneto-elastic problems in electrical machines: Proc. Int. Conf. Electric Machines and Drives IEMD'99.

[13] Belahcen, A. (2005) Magneto-elastic coupling in rotating electrical machines: IEEE Trans Magn 41 1624-1627.

[14] Rasilo, P., Aydin, U., Singh, D., Martin, F., Kouhia; R., Belahcen, A., and Arkkio, A. (2016) Multiaxial magnetomechanical modelling of electrical machines with hysteresis: 8 th
IET International Conference on Power Electronics, Machines and Drives (PEMD 2016) Glasgow 1-6.

[15] Ionel, D.M., Popescu, M., McGilp, M.I., Miller, T.J.E., Dellinger, S.J., and Heideman, R.J. (2007) Computation of core losses in electrical machines using improved models for laminated steel: IEEE Trans Ind Appl 43 1554-1564.

[16] Gracia, M.H., Lange, E., and Hameyer, K. (2007) Numerical calculation of iron losses in electrical machines with a modified post-processing formula: Proceedings of the 16th International Symposium on Electromagnetic Fields COMPUMAG, Aachen, Germany.

[17] Belahcen, A., Dlala, E., Fonteyn, K., and Belkasim, M. (2010) A posteriori iron loss computation with a vector hysteresis model: COMPEL - The International Journal for Computation and Mathematics in Electrical and Electronic Engineering 29 1493-1503.

[18] Sablik, M.J., and Jiles, D.C. (1993) Coupled magnetoelastic theory of magnetic and magnetostrictive hysteresis: IEEE Trans Magn 29 2113-2123.

[19] Suzuki, T., and Matsumoto, E. (2005) Comparison of JilesAtherton and Preisach models extended to stress dependence in magnetoelastic behaviours of a ferromagnetic material: $J$ Mat Procs Tech 161 141-145.

[20] Daniel, L., Rekik, M., and Hubert, O. (2014) A multiscale model for magneto-elastic behaviour including hysteresis effects: Arch Appl Mech 84 1307-1323.

[21] Daniel, L., Hubert, O., and Rekik, M. (2015) A simplified 3D constitutive law for magneto-mechanical behavior: IEEE Trans Magn 517300704 (4pp).

[22] Rasilo, P., Singh, D., Aydin, U., Martin, F., Kouhia, R., Belahcen, A., and Arkkio, A. (2016) Modeling of hysteresis losses in ferromagnetic laminations under mechanical stress: IEEE Trans Magn 527300204 (4pp).

[23] Bertotti, G. (1988) General properties of power losses in soft ferromagnetic materials: IEEE Trans Magn 24 621-630.

[24] Ionel, D.M. (2006) On the variation with flux and frequency of the core loss coefficients in electrical machines: IEEE Trans Ind Appl 42 658-667.

[25] Krings, A. and Soulard, J. (2010) Overview and comparison of iron loss models for electrical machines: 5th International Conference and Exhibition on Ecological Vehicles and Renewable Energies (EVER 10) (Monte-Carlo- MONACO).

[26] Steentjes, S., Leßmann, M., and Hameyer, K. (2012) Advanced iron-loss calculation as a basis for efficiency improvement of electrical machines in automotive application: Electrical Systems for Aircraft, Railway and Ship Propulsion (ESARS2012), Bologna, 1-6.

[27] Steentjes, S., Pfingsten, G., Hombitzer, M., and Hameyer, K. (2013) Iron-Loss model with consideration of minor loops applied to FE-simulations of electrical machines: IEEE Trans Magn 49 3945-3948.

[28] Ali, K., Atallah, K., and Howe, D. (1997) Prediction of mechanical stress effects on the iron loss in electrical machines: J Appl Phy 81 4119-4121.

[29] Permiakov, V., Dupré, L., Pulnikov, A., and Melkebeek, J. (2004) Loss separation and parameters for hysteresis modelling under compressive and tensile stresses: J Magn Magn Mater 272-276 e553-e554. 
[30] Saeed, O., Saleem, A., Rahman, T., Chromik, R., and Lowther, D. (2015) Iron loss models under static stress for non-oriented and grain-oriented steel: 20th International Conference on the Computation of Electromagnetic Fields (Compumag-2015), Montreal, Quebec, Canada.

[31] Singh, D., Rasilo, P., Martin, F., Belahcen, A., and Arkkio, A. (2015) Effect of mechanical stress on excess loss of electrical steel sheets: IEEE Trans Magn 511001204 (4pp).

[32] Karthaus, J., Steentjes, S., Leuning, N., and Hameyer, K. (2017) Effect of mechanical stress on different iron loss components up to high frequencies and magnetic flux densities: COMPEL - The international Journal for Computation and Mathematics in Electrical and Electronic Engineering 36 580-592.

[33] Hargreaves, P.A., Mecrow, B.C., and Hall, R. (2011) Calculation of iron losses in electrical generators using finite element analysis: IEEE International Electrical machines \& Derives Conference (IEMDC-2011), pp. 1368-1373.

[34] Zirka, S.E., Moroz, Y.I., Marketos, P., Moses, A.J., Jiles, D.C., and Matsuo, T. (2008) Generalization of the classical method for calculating dynamic hysteresis loops in grain-oriented electrical steels: IEEE Trans Magn 44 2113-2126.

[35] Bertotti, G. 1998 Hysteresis in Magnetism (San Diego, CA: Academic).

[36] Rahman, T., Akiror, J.C., Pillay, P., and Lowther, D.A. (2013) Comparison of iron loss prediction formulae, 19th International Conference on the Computation of Electromagnetic Fields COMPUMAG-2013, Budapest, Hungary.

[37] Jiles,D. C. and Atherton, D.L. (1986) Theory of ferromagnetic hysteresis: J Magn Magn Mater 61 48-60.

[38] Chen, Y., and Pillay, P., (2002) An improved fromula for lamination core loss calculations in machines operating with high frequency and high flux density excitation: IEEE 37th IAS Annual Meeting Conference, pp. 759-766.

[39] Kai, Y., Tsuchida, Y., Todaka, T., and Enokizono, M. (2014) Influence of biaxial stress on vector magnetic properties and 2D magnetostriction of a non-oriented electrical steel sheet under alternating magnetic flux conditions: IEEE Trans Magn 50 6100204 (4pp).

[40] Rekik, M., Hubert, O., and Daniel, L. (2014) Influence of a multiaxial stress on the reversible and irreversible magnetic behavior of 3\% Si-Fe alloy: Int J App Electmags Mech 44 301315.

[41] Aydin, U., Rasilo, P., Martin, F., Belahcen, A., Daniel, L. Haavisto, A., Arkkio, A. . (2019) Effect of multi-axial stress on iron losses of electrical steel sheets, J Magn Magn Mater 469 19-27.

[42] Yamazaki, K., Mukaiyama, H., and Daniel, L. (2018) Effect of multi-axial mechanical stress on loss characteristics of electrical steel sheets and interior permanent magnet machines: IEEE Trans Magn 541300304 (4pp).

[43] Aydin, U., Rasilo, P., Martin, F., Singh, D., Daniel, L., Belahcen, A., Kouhia, R., and Arkkio, A. (2017) Modelling the effect of multiaxial stress on magnetic hysteresis of electrical steel sheets: a comparison: IEEE Trans Magn 532000904 (4pp). 